\title{
EXTREMAL PROPERTIES OF THE FUNCTIONALS CONNECTED WITH THE SYSTEMS OF CONSERVATION LAWS
}

\author{
YU. G. RYKOV* \\ Keldysh Institute of Applied Mathematics, RAS. Moscow, Russia \\ *Corresponding author. E-mail: yu-rykov@yandex.ru
}

DOI: $10.20948 /$ mathmontis-2019-46-3

Summary. The paper contains a further concretization of the variational approach to the theory of systems of conservation laws described in the earlier author's work. This approach involves the development of methods for proving the existence and uniqueness theorems for generalized solutions that are based on the search for critical points of functionals in Banach spaces. A new definition of the generalized solution is proposed and its equivalence to the traditional one for the functions of a simple structure is proved. A new strategy for proving existence and uniqueness theorems is proposed. A number of illustrative theorems outlining the implementation of this strategy are proved.

\section{INTRODUCTION}

The paper is devoted to further assessment of new approach to the theory of systems of conservation laws, which was proposed in [1]. In order to give brief impression why any new approach in the field of conservation laws seems to be necessary, we give below several guidelines, these guidelines in no way pretend to reflect even a main topics in an extensive literature on the conservation laws theory. As is known, the state of the art in the field of quasilinear hyperbolic conservation laws systems is far from completeness. Still a sufficiently complete theory has been constructed only for a single conservation law by S.N.Kruzhkov in 1970, [2]. In the case of systems, fairly general results have been obtained only for one spatial variable and, as a rule, under the assumption that the variation range of the unknown functions is small, see basic research [3] and later interpretation in, for example, [4]. In the framework of present paper we set aside much more complex issues connected with the multidimensional case and do not mention the corresponding researches and results. For one dimensional systems, in order to remove smallness constraints, the vanishing viscosity method was used. This method leads to excellent results in the case of one conservation law (Kruzhkov theory) and in the case of systems it leads to the notion of measure-valued solutions [5]. Further the general existence theorems for generalized solutions to systems of two hyperbolic conservation laws (one spatial variable) with the aid of compensated compactness principle were obtained. But then the vanishing viscosity method seems to become the stumbling block for the theory because of the lack of necessary apriori estimates when the number of equations equals or more than three.

Moreover there are the facts that can be interpreted in the sense that the vanishing viscosity method, may be, is not very relevant to study the quasilinear systems of hyperbolic conservation laws. First, certain systems of two conservation laws, which are strictly hyperbolic, genuinely nonlinear have no classical solutions to Riemann problem and the application of viscosity method gives the emergence of delta-functions along the shock lines [6]. This seems inappropriate because there is no satisfactory interpretation how to deal with

2010 Mathematics Subject Classification: 35L65, 35A15, 58E30.

Key words and Phrases: Conservation laws, Generalized solutions, Variational approach, Critical points of the functionals, Palais-Smale condition. 
delta-functions in nonlinear problems. The example of such systems considered in [6] is shown below

$$
\begin{gathered}
u_{t}+\left(u^{2}-v\right)_{x}=0 \\
v_{t}+\left(u^{3} / 3-u\right)_{x}=0,
\end{gathered}
$$

here $u=u(t, x), v=v(t, x)$ and $(t, x) \in \mathbb{R}^{+} \times \mathbb{R}$, the subscripts $t$ and $x$ denote the corresponding partial derivatives. Moreover, the system (1) can be obtained from the system of isothermal gas dynamics via certain nonlinear transformation.

In addition the recent paper by D.Serr [7] studies the so-called divergence-free positive symmetric tensors (DPT) and their connection with the fluid dynamics. The DPT is locally integrable tensor $T(y), y=\left(y_{1}, \ldots, y_{m}\right), T=\left\{T_{i j}, i=1, \ldots, n ; j=1, \ldots, m\right\}$, which is positive definite or positive semi-definite and $\operatorname{Div} T \equiv\left(\sum_{j=1}^{m} \partial T_{i j} / \partial y_{j}\right)_{1 \leq i \leq n}=0$. The divergence is understood in the weak sense. Many physical systems can be put in such a form including Euler and Navier-Stokes systems. The main result of [7] is the discovery of new apriori estimate for DPT, namely the finiteness of integral

$$
\int(\operatorname{det} T(y))^{1 /(m-1)} d y \leq\left(\operatorname{det} \int T(y) d y\right)^{1 /(m-1)} .
$$

In case of multidimensional gas dynamics which is considered as the one of most important examples of conservation laws systems the estimate (2) yields the following estimate

$$
\int_{0}^{T} d t \int_{\mathbb{R}^{m}} \rho^{1 / m} P d y \leq \text { const }
$$

where $\rho$ is the density and $P$ is the pressure. The estimates like (3) rule out the possibility of delta-shocks as in [6], which are obtained via vanishing viscosity method. Moreover, as it is highlighted in [7] though the Navier-Stokes system has the form of divergent-free tensor it lacks positivity and thus in general cannot provide estimates like (3). So [7] asserts that vanishing viscosity method seems not suitable but the construction of a generalized solution should involve an approximation process which is consistent with the estimate (3), i.e. for the Euler equation the Boltzmann equation approximation or numerical schemes are proposed in [7].

Taking into account the fact that abovementioned methods are known for a long time but their application still seems does not lead to satisfactory constructions, in the present paper we discus another possibility (see [1]) of approaching the notion of generalized solutions, which is different from traditional approaches. Namely, we strive developing the notion of generalized solution, which is based on the existence of critical points of functionals in Banach space. As it can be seen below this task in fact requires much investigation and therefore here we only establish the general framework for this new approach.

The paper is organized as follows. In section 2 we recall the very basic known definitions and facts on the quasilinear conservation laws theory and introduce variational interpretation 
of these concepts in lines with [1]. Also we give certain new interpretation of some results from [1] that are relevant to the topic of the present paper. In section 3 a new strategy for proving existence and uniqueness theorems is proposed. A number of illustrative theorems outlining the implementation of this strategy are proved though these theorems at the moment are still far from the proof of any existence and uniqueness theorem. Finally in section 4 several concluding remarks are made.

\section{A VARIATIONAL VIEW ON THE SYSTEMS OF CONSERVATION LAWS}

Consider the Cauchy problem for the system of quasilinear conservation laws, which is assumed to be strictly hyperbolic

$$
\boldsymbol{U}_{t}+\boldsymbol{F}(\boldsymbol{U})_{x}=0 \quad, \quad \boldsymbol{U}(0, x)=\boldsymbol{U}_{0}(x),
$$

where $\quad(t, x) \in \Pi_{T} \equiv\{(t, x):(t, x) \in[0, T] \times \mathbb{R}\}, \quad \boldsymbol{U}(t, x)=\left(u_{1}(t, x), \ldots, u_{n}(t, x)\right) \quad$ and $\boldsymbol{F}=\left(f_{1}, \ldots, f_{n}\right)$ is a sufficiently smooth (at least of class $\left.C^{1}\left(\mathbb{R}^{n}\right)\right)$ vector function of variables $\left(u_{1}, \ldots, u_{n}\right)$. The solutions to system (4) that take given initial values are understood in the generalized sense with respect to the following conventional definition.

DEFINITION 1. Let $\boldsymbol{U}_{0}(x) \in \mathbb{R}^{n}$ be a bounded measurable function. A bounded and measurable function $\boldsymbol{U}(t, x)$ in $\Pi_{T}$ is called a generalized solution to the problem (4) if, for every test function $\varphi \in C^{\infty}([0, T) \times \mathbb{R})$ such that $\varphi \in C_{0}^{\infty}(\mathbb{R})$ for fixed $t \in[0, T]$ and $\varphi \equiv 0$ for $T_{1} \leq t \leq T, T_{1}<T$, the following integral identity holds:

$$
\iint_{\Pi_{T}}\left[\boldsymbol{U} \varphi_{t}+\boldsymbol{F}(\boldsymbol{U}) \varphi_{x}\right] d x d t+\int_{\mathbb{R}} \boldsymbol{U}_{0} \varphi(0, x) d x=0 .
$$

It is well known that the relations (5) do not guarantee the uniqueness of a solution to the problem (4). Thus an additional conditions are required for the function $\boldsymbol{U}(t, x)$. In the modern literature it is believed that such conditions should have the form of entropy inequalities (and this is true at least for the unknown functions with apriori small variation range).

DEFINITION 2. Let us call convex positive function $\eta(\boldsymbol{U}) \in C^{1}\left(\mathbb{R}^{n}\right)$ an entropy for the system in (4) if for the classical solutions an additional conservation law holds

$$
\eta(\boldsymbol{U}(t, \boldsymbol{x}))_{t}+(q(\boldsymbol{U}(t, \boldsymbol{x})))_{x}=0,
$$

providing certain smooth enough flow function $q\left(u_{1}, \ldots, u_{n}\right)$.

DEFINITION 3. The function $\boldsymbol{U}(t, x)$, which is the generalized solution to (4) in the sense of Definition 1, will be called an entropy solution to the problem (4) if for every entropy $\eta(\boldsymbol{U})$ from the Definition 2 and test function $\varphi(t, x) \geq 0$ from the Definition 1 the following inequality holds 


$$
\iint_{\Pi_{T}}\left[\eta(\boldsymbol{U}) \varphi_{t}+q(\boldsymbol{U}) \varphi_{x}\right] d x d t+\int_{\mathbb{R}} \eta\left(\boldsymbol{U}_{0}\right) \varphi(0, x) d x \geq 0 .
$$

In the present paper we will not touch the questions of uniqueness, certain interpretation of the notion of entropy solution from the variational point of view (see just below) can be found in [1]. Here we are interested in the non-conventional procedure of seeking any weak solution to system (4).

The main idea of variational approach that has been developed to a certain degree in [1] is as follows. Let us consider instead of function $\boldsymbol{U}(t, x)$ the functional $\boldsymbol{J}: \chi(\tau) \in C^{1}([0, T], \mathbb{R}) \rightarrow \mathbb{R}^{n}$,

$$
\boldsymbol{J} \equiv \int_{0}^{T} \boldsymbol{L}(\dot{\chi}, \boldsymbol{U}) d \tau ; \boldsymbol{L}(\dot{\chi}, \boldsymbol{U}) \equiv \boldsymbol{U}(\tau, \chi(\tau)) \dot{\chi}(\tau)-\boldsymbol{F} \circ \boldsymbol{U}(\tau, \chi(\tau))
$$

It is shown in [1] that under certain regularity restrictions to $\boldsymbol{U}(t, x)$ the condition $\delta \boldsymbol{J}=0$ along some trajectory $x=\chi_{\text {extr }}(t)$ implies that $\boldsymbol{U}(t, x)$ satisfies (4) in classical sense along $x=\chi_{\text {extr }}(t)$ where $\boldsymbol{U}(t, x)$ is smooth and at the points of intersection of $x=\chi_{\text {extr }}(t)$ with discontinuities of $\boldsymbol{U}(t, x)$ the Hugoniot relations hold. This means that having the aim of seeking the generalized solutions to (4) it could be useful to consider instead of functions $\boldsymbol{U}(t, x)$ the functionals $\boldsymbol{J}$, and study the extremal properties of such functionals.

In order to further assess this idea we assume that $\boldsymbol{U}(t, x)$ belongs to the class $K$ of piecewise continuously differential functions with finite number of discontinuities. Such a class was used by O.Oleinik in [8] and it is useful for the initial stages of construction of theories connected with the systems of conservation laws (see also [1]). More precisely in [1], in particular, the following theorem was proved.

THEOREM 4. Let $\boldsymbol{U}(t, x) \in K$, and suppose that there exists a trajectory $\chi_{\text {extr }}(t) \in C^{1}([0, T], \mathbb{R})$ such that $\delta \boldsymbol{J}=0$ for this trajectory. Then, at the points $x=\chi_{\text {extr }}(t)$ where $\boldsymbol{U}(t, x)$ is smooth, equations (4) hold in the classical sense, and at the points of intersection of $\chi_{\text {extr }}(t)$ with the discontinuity lines of the function $\boldsymbol{U}(t, x)$, the Hugoniot relations

$$
\frac{d s}{d t} \cdot\left(\boldsymbol{U}^{+}-\boldsymbol{U}^{-}\right)=\boldsymbol{F}\left(\boldsymbol{U}^{+}\right)-\boldsymbol{F}\left(\boldsymbol{U}^{-}\right)
$$

are satisfied; here $x=s(t)$ is the discontinuity curve and $\boldsymbol{U}^{ \pm} \equiv \boldsymbol{U}(t, s(t) \pm 0)$. Moreover, the expression for $\delta^{2} \boldsymbol{J}$ on the trajectory $x=\chi_{\text {extr }}(t)$ contains only terms depending on $(\delta \chi)^{2}$ (i.e. the quadratic form does not contain terms with $\delta \dot{\chi}$ ).

The Theorem 4 shows that the system (4) is 'fulfilled in generalized sense' along the extremal trajectories of $\boldsymbol{J}$ and locally in $(t, x)$ the extremum in general is either maximum or 
minimum with respect to C-metrics. This means that the functional $\boldsymbol{J}$, which corresponds to the generalized solution of (4), is the functional, for which any trajectory is extremal. Thus we can consider the set of all functionals of type (8) and try to find the one or ones with the property just described. This view constitutes a variational approach and potential strategy for finding the generalized solution to (4).

In the present paper we extend such a view as follows. Introduce the function $\boldsymbol{V}(t, x) \equiv \int \boldsymbol{U}(t, p) d p$ and consider the functional $\boldsymbol{I}: \chi(\tau) \in C([0, T], \mathbb{R}) \rightarrow \mathbb{R}^{n}$ instead of $\boldsymbol{J}$

$$
\boldsymbol{I} \equiv \int_{0}^{T} \boldsymbol{M}(\boldsymbol{U})(\tau, \chi(\tau)) d \tau \equiv \int_{0}^{T}\left[\frac{\partial \boldsymbol{V}}{\partial \tau}(\tau, \chi(\tau))+\boldsymbol{F} \circ \boldsymbol{U}(\tau, \chi(\tau))\right] d \tau .
$$

With the provisions of functional (10) we can notice that it, in general, acts on the space of only continuous functions $\chi(\tau)$.

THEOREM 5. Let $\boldsymbol{U}(t, x) \in K$, and suppose that there exists a trajectory $\chi_{\text {extr }}(t) \in C([0, T], \mathbb{R}), \chi_{\text {extr }}(0)=y, \chi_{\text {extr }}(T)=X$ such that $\delta \boldsymbol{I}$ is defined at $\chi_{\text {extr }}(t)$ (i.e., in particular, $\left.\chi_{\text {extr }}(t) \in C^{1}([0, T], \mathbb{R})\right)$ and $\delta \boldsymbol{I}=0$ for this trajectory. Then, at the points $x=\chi_{\text {extr }}(t)$ where $\boldsymbol{U}(t, x)$ is smooth, equations (4) hold in the classical sense, and at the points of intersection of $\chi_{\text {extr }}(t)$ with the discontinuity lines of the function $\boldsymbol{U}(t, x)$, the Hugoniot relations (9) are satisfied. Moreover, the property $\delta \boldsymbol{I}=0$ means that the function $\boldsymbol{M}(\boldsymbol{U})$ is continuous along discontinuities of $\boldsymbol{U}(t, x)$ and value of $\delta^{2} \boldsymbol{I}$ changes by the jump of $\boldsymbol{M}(\boldsymbol{U})_{\tau}$.

PROOF. Because of the structure of class $\mathrm{K}$ for the proof of the theorem 5 it is enough to consider the situation when function $\boldsymbol{U}(t, x)$ has the single discontinuity line $x=s(t) \in C^{1}([0, T])$ and there exists only one point of intersection of $x=s(t)$ and $x=\chi_{\text {extr }}(t)$. Let us note that in this case, as it is shown below, $\delta \boldsymbol{I}$ is defined if $\dot{\chi}_{\text {extr }}(t) \neq \dot{s}(t)$ at the intersection point of $x=s(t)$ and $x=\chi_{\text {extr }}(t)$. Further the subscript 'extr' will be omitted for notations' simplicity. We have

$$
\delta \boldsymbol{I}=\left.\left.\frac{d}{d \alpha}\right|_{\alpha=0} \int_{0}^{T} \boldsymbol{M}(\boldsymbol{U})_{\chi+\alpha h} d \tau \equiv \frac{d}{d \alpha}\right|_{\alpha=0} \int_{0}^{T}\left[\frac{\partial \boldsymbol{V}}{\partial \tau}+\boldsymbol{F} \circ \boldsymbol{U}\right](\tau, \chi(\tau)+\alpha h(\tau)) d \tau,
$$

and

$$
\delta^{2} \boldsymbol{I}=\left.\left.\left.\frac{d^{2}}{d \alpha^{2}}\right|_{\alpha=0} \int_{0}^{T} \boldsymbol{M}(\boldsymbol{U})\right|_{\chi+\alpha h} d \tau \equiv \frac{d^{2}}{d \alpha^{2}}\right|_{\alpha=0} \int_{0}^{T}\left[\frac{\partial \boldsymbol{V}}{\partial \tau}+\boldsymbol{F} \circ \boldsymbol{U}\right](\tau, \chi(\tau)+\alpha h(\tau)) d \tau,
$$

where $h(0)=h(T)=0$.

Suppose that $\tau_{0}$ is such moment of time that $\chi\left(\tau_{0}\right)=s\left(\tau_{0}\right)$ and consequently $\tau^{*}(\alpha)$ is the time moment where $\chi\left(\tau^{*}\right)+\alpha h\left(\tau^{*}\right)=s\left(\tau^{*}\right)$. Thus as $0 \leq \tau \leq \tau^{*}(\alpha)$ the function 
$\boldsymbol{M}(\boldsymbol{U})(\tau, \chi(\tau))$ is smooth and will be denoted as $\boldsymbol{M}\left(\boldsymbol{U}^{-}\right)$; at $\tau=\tau^{*}(\alpha)$ the function $\boldsymbol{M}(\boldsymbol{U})$ has the discontinuity; and as $\tau^{*}(\alpha) \leq \tau \leq T$ the function $\boldsymbol{M}(\boldsymbol{U})(\tau, \chi(\tau))$ is also sooth and will be denoted by $\boldsymbol{M}\left(\boldsymbol{U}^{+}\right)$. Now we split the integrals in (11), (12) by the integrals with respect to the segments $\left[0, \tau^{*}(\alpha)\right],\left[\tau^{*}(\alpha), T\right]$, perform the differentiation as in (11), (12) and obtain for $\delta \boldsymbol{I}$

$$
\begin{aligned}
& \delta \boldsymbol{I}=\int_{0}^{\tau_{0}} \boldsymbol{M}\left(\boldsymbol{U}^{-}\right)_{\chi}(\tau, \chi(\tau)) h(\tau) d \tau+\int_{\tau_{0}}^{T} \boldsymbol{M}\left(\boldsymbol{U}^{+}\right)_{\chi}(\tau, \chi(\tau)) h(\tau) d \tau+ \\
& \left.\tau_{\alpha}^{*}(0)\left[\boldsymbol{M}\left(\boldsymbol{U}^{-}\right)-\boldsymbol{M}\left(\boldsymbol{U}^{+}\right)\right]\right|_{\tau=\tau_{0}},
\end{aligned}
$$

and for $\delta^{2} \boldsymbol{I}$

$$
\begin{aligned}
& \delta^{2} \boldsymbol{I}=\int_{0}^{\tau_{0}} \boldsymbol{M}\left(\boldsymbol{U}^{-}\right)_{x x}(\tau, \chi(\tau)) h^{2}(\tau) d \tau+\int_{\tau_{0}}^{T} \boldsymbol{M}\left(\boldsymbol{U}^{+}\right)_{x x}(\tau, \chi(\tau)) h^{2}(\tau) d \tau+ \\
& \left.\tau_{\alpha \alpha}^{*}(0)\left[\boldsymbol{M}\left(\boldsymbol{U}^{-}\right)-\boldsymbol{M}\left(\boldsymbol{U}^{+}\right)\right]\right|_{\tau=\tau_{0}}+\left.2 \tau_{\alpha}^{*}(0) h\left(\tau_{0}\right)\left[\boldsymbol{M}\left(\boldsymbol{U}^{-}\right)_{x}-\boldsymbol{M}\left(\boldsymbol{U}^{+}\right)_{x}\right]\right|_{\tau=\tau_{0}}+ \\
& \left.\tau_{\alpha}^{*}(0)^{2}\left[\boldsymbol{M}\left(\boldsymbol{U}^{-}\right)_{\tau}-\boldsymbol{M}\left(\boldsymbol{U}^{+}\right)_{\tau}+\dot{\chi}\left(\tau_{0}\right)\left(\boldsymbol{M}\left(\boldsymbol{U}^{-}\right)_{x}-\boldsymbol{M}\left(\boldsymbol{U}^{+}\right)\right)\right]\right|_{\tau=\tau_{0}} .
\end{aligned}
$$

It is also easy to check that $\tau_{\alpha}^{*}(0)=\left.\frac{h}{\dot{s}-\dot{\chi}}\right|_{\tau=\tau_{0}}$ and $\tau_{\alpha \alpha}^{*}(0)=\left.\frac{(\ddot{\chi}-\ddot{s}) \tau_{\alpha}^{*}(0)^{2}+2 \dot{h} \tau_{\alpha}^{*}(0)}{\dot{s}-\dot{\chi}}\right|_{\tau=\tau_{0}}$.

From (13) it follows that if $\delta \boldsymbol{I}=0$ then

$$
\boldsymbol{M}\left(\boldsymbol{U}^{ \pm}\right)_{x}=0,\left.\left[\boldsymbol{M}\left(\boldsymbol{U}^{-}\right)-\boldsymbol{M}\left(\boldsymbol{U}^{+}\right)\right]\right|_{\tau=\tau_{0}}=0
$$

because the $h(\tau)$ is arbitrary. Let us note that if $\boldsymbol{U}(t, x) \in K$ then $\boldsymbol{V}(t, x)$ is continuous and $\boldsymbol{V}^{-}(\tau, s(\tau))=\boldsymbol{V}^{+}(\tau, s(\tau)) \quad$ for $\quad$ any $\quad \tau \in[0, T] \quad$ where $\quad x=s(\tau) \quad$ exists. Thus $\boldsymbol{V}_{\tau}^{-}-\boldsymbol{V}_{\tau}^{+}=\dot{s}\left(\boldsymbol{V}_{x}^{+}-\boldsymbol{V}_{x}^{-}\right)=\dot{s}\left(\boldsymbol{U}^{+}-\boldsymbol{U}^{-}\right)$. Therefore from (15) we obtain

$$
\begin{aligned}
& \left.0=\boldsymbol{M}\left(\boldsymbol{U}^{ \pm}\right)_{x}=\left[\frac{\partial \boldsymbol{V}^{ \pm}}{\partial \tau}+\boldsymbol{F} \circ \boldsymbol{U}^{ \pm}\right]\right]_{x}(\tau, \chi(\tau))=\boldsymbol{U}_{\tau}^{ \pm}+\boldsymbol{F} \circ \boldsymbol{U}_{x}^{ \pm}(\tau, \chi(\tau)), \\
& 0=\left.\left[\boldsymbol{M}\left(\boldsymbol{U}^{-}\right)-\boldsymbol{M}\left(\boldsymbol{U}^{+}\right)\right]\right|_{\tau=\tau_{0}}=\left.\left[\boldsymbol{V}_{\tau}^{-}-\boldsymbol{V}_{\tau}^{+}+\boldsymbol{F} \circ \boldsymbol{U}^{-}-\boldsymbol{F} \circ \boldsymbol{U}^{+}\right]\right|_{\tau=\tau_{0}}= \\
& {\left.\left[\dot{s}\left(\boldsymbol{U}^{+}-\boldsymbol{U}^{-}\right)+\boldsymbol{F} \circ \boldsymbol{U}^{-}-\boldsymbol{F} \circ \boldsymbol{U}^{+}\right]\right|_{\tau=\tau_{0}},}
\end{aligned}
$$

and we've got the assertion of Theorem 5 concerning the $\delta \boldsymbol{I}=0$ property.

Finally, substituting expressions (15) into the formula (16) for $\delta^{2} \boldsymbol{I}$ it can be seen that if $\delta \mathbf{I}=0$ then 


$$
\begin{aligned}
& \delta^{2} \boldsymbol{I}=\int_{0}^{\tau_{0}} \boldsymbol{M}\left(\boldsymbol{U}^{-}\right)_{x x}(\tau, \chi(\tau)) h^{2}(\tau) d \tau+\int_{\tau_{0}}^{T} \boldsymbol{M}\left(\boldsymbol{U}^{+}\right)_{x x}(\tau, \chi(\tau)) h^{2}(\tau) d \tau+ \\
& \left.\tau_{\alpha}^{*}(0)^{2}\left[\boldsymbol{M}\left(\boldsymbol{U}^{-}\right)_{\tau}-\boldsymbol{M}\left(\boldsymbol{U}^{+}\right)_{\tau}\right]\right|_{\tau=\tau_{0}} .
\end{aligned}
$$

Thus the theorem 5 is proved.

It is easy to check that the following relation is true $\boldsymbol{I}+\boldsymbol{J}=\int^{X} \boldsymbol{U}(T, p) d p-\int^{y} \boldsymbol{U}(0, p) d p$. Thus the results of theorem 5 clarifies the variational properties of $\boldsymbol{J}$ and demonstrates the equivalence of introducing $\boldsymbol{J}$ or $\boldsymbol{I}$ for the functions $\boldsymbol{U}(t, x) \in K$. Further, it is possible to lay into the basis of the definition of generalized solution the variational properties of functional $\boldsymbol{J}$ (but also use $\boldsymbol{I}$ for technical reasons), i.e the function $\boldsymbol{U}(t, x)$ belonging to one or the other functional space will be the generalized solution if any $C^{1}$ trajectory for corresponding $\boldsymbol{J}$ is its critical point. In the next section this approach will be put in more rigorous frame.

\section{A NEW STRATEGY FOR FINDING THE GENERALIZED SOLUTION}

Let us start with the variational definition of generalized solution to the system of conservation laws (4). We remind that in the present paper we do not touch the question of satisfaction with the initial conditions and the question of uniqueness.

Consider the vector function $\boldsymbol{U}(t, x) \in B$, where $B$ is some functional Banach space (for example, $B V, L_{1}, L_{1, l o c}, L^{\infty}$, etc.). Also consider the set $\mathrm{X}$ of all trajectories $\chi(t) \in C^{1}([0, T], \mathbb{R})$. Let $\Gamma_{U} \subset \mathrm{X}$ be the set of trajectories (depending on considered function $\boldsymbol{U}$ ) where the integral $\boldsymbol{J}$, see (8), is well defined. Denote by $\Delta_{U} \subset \Gamma_{U}$ the set of trajectories where $\delta \boldsymbol{J}$ exists.

DEFINITION 6. Consider the vector function $\boldsymbol{U}(t, x) \in B$, where $B$ is some functional Banach space. Consider the functional $\boldsymbol{J}: \chi(\tau) \in C^{1}([0, T], \mathbb{R}) \cap \Gamma_{U} \rightarrow \mathbb{R}^{n}$ with respect to expression (8). The function $\boldsymbol{U}(t, x)$ will be called the generalized solution to conservation laws system (4) if any trajectory $\chi(\tau) \in C^{1}([0, T], \mathbb{R}) \cap \Gamma_{U} \cap \Delta_{U}$ is critical for $\boldsymbol{J}$ (i.e. $\delta \boldsymbol{J}=0)$.

Let us first show that there are sufficiently many trajectories where $\boldsymbol{J}$ is defined for locally integrable function $\boldsymbol{U}(t, x)$.

THEOREM 7. Consider any diffeomorphism of $\mathbb{R}^{+} \times \mathbb{R}$ having the form: $t^{\prime}=t, x^{\prime}=\xi(t, x), \quad \xi_{x} \neq 0$. Suppose $\boldsymbol{U}(t, x) \in L_{1, l o c}\left(\mathbb{R}^{+} \times \mathbb{R}\right) \quad$ with respect to Lebesgue measure. Then for a.e. $x$ the function $\boldsymbol{U}(t, \xi(t, x))$ is locally integrable with respect to $t$ and the functional $\boldsymbol{J}$ is defined provided the following estimate holds $|\boldsymbol{F}(\boldsymbol{U})| \leq|\boldsymbol{U}|, \boldsymbol{U} \in \mathbb{R}^{n}$. 
PROOF. Let us take the diffeomorphism $\left(t^{\prime}, x^{\prime}\right)=\psi(t, x)$ in the form pointed out in the conditions of theorem 7 . For any measurable set $A$ the set $\psi(A)$ is also measurable and the change of variables formula is true

$$
\iint_{\psi(A)} \boldsymbol{U}\left(t^{\prime}, x^{\prime}\right) d t^{\prime} d x^{\prime}=\iint_{A} \boldsymbol{U} \circ \psi(t, x) \frac{\partial\left(t^{\prime}, x^{\prime}\right)}{\partial(t, x)} d t d x .
$$

At this, the function under the right hand side integral in (18) is integrable. By the Fubini theorem for a.e. $x$ the function $\boldsymbol{U}(t, \xi(t, x)) \xi_{x}$ is locally integrable with respect to $t$ and so does the function $\boldsymbol{U}(t, \xi(t, x))$ because $\xi_{x} \neq 0$. Taking into account the estimate on $\boldsymbol{F}(\boldsymbol{U})$ from the conditions of theorem 7 we get the definiteness of functional $\boldsymbol{J}$. The theorem is proved.

The next theorem demonstrates another way of investigation the relevance of definition 6 .

THEOREM 8. Suppose $\boldsymbol{U}(t, x) \in B V\left(\mathbb{R}^{+} \times \mathbb{R}\right)$ and is a generalized solution of system (4) in the sense of definition 6. Suppose there exists the following set of trajectories $\Omega_{U}: x=\xi(t, \alpha, \varepsilon) \equiv \alpha \chi_{1}(t)+(1-\alpha) \chi_{2}(t, \varepsilon), \alpha \in[0,1], \chi_{1}(t)<\chi_{2}(t, \varepsilon) \in C^{1}([0, T], \mathbb{R})$ and $\chi_{2}(t)=\chi_{1}(t)+\varepsilon$ for $t \in\left[0, \tau_{1}\right] \cup\left[\tau_{2}, T\right], \tau_{2}>\tau_{1}>0 ; \varepsilon>0$ is small. At this let $\boldsymbol{J}$ and $\delta \boldsymbol{J}$ are defined for the trajectories from $\Omega_{U}$ and $\delta \boldsymbol{J}=0$ for any trajectory. Then $\iint_{E}\left(\boldsymbol{U}_{t}+\boldsymbol{F}(\boldsymbol{U})_{x}\right) d t d x=0$ in the sense of measures, $E: \tau_{1} \leq t \leq \tau_{2}, \chi_{1}(t) \leq x \leq \chi_{2}(t, 0)$.

PROOF. Consider $\boldsymbol{J}$ along the trajectories from $\Omega_{U}$ as the function of $\alpha$ with fixed $\varepsilon$. Thus we have

$$
\boldsymbol{J}(\alpha)_{\varepsilon}=\int_{0}^{T}\left[\boldsymbol{U}(\tau, \xi(\tau, \alpha, \varepsilon)) \xi_{\tau}(\tau, \alpha, \varepsilon)-\boldsymbol{F} \circ \boldsymbol{U}(\tau, \xi(\tau, \alpha, \varepsilon))\right] d \tau .
$$

Further, according to the conditions of the theorem $\delta \boldsymbol{J}$ exists for trajectories from $\Omega_{U}$ and therefore $\boldsymbol{J}_{\varepsilon}(\alpha)$ is differentiable for any small $\varepsilon>0$. By mean value theorem $\boldsymbol{J}_{\varepsilon}(1)-\boldsymbol{J}_{\varepsilon}(0)=\delta \boldsymbol{J}_{\varepsilon}(\bar{\alpha}), 0<\bar{\alpha}<1$. But $\delta \boldsymbol{J}$ vanishes for all trajectories from $\Omega_{\boldsymbol{U}}$, hence $\boldsymbol{J}_{\varepsilon}(1)=\boldsymbol{J}_{\varepsilon}(0)$ for any small $\varepsilon>0$. Passing to the limit as $\varepsilon \rightarrow 0$ we immediately have from (19)

$$
\int_{\partial E}[\boldsymbol{U}(t, x) d x-\boldsymbol{F} \circ \boldsymbol{U}(t, x) d t]=0 .
$$

For $B V$ functions Green formula is valid and the assertion of the theorem follows from (20), see also [9]. The theorem is proved.

REMARK 9. In case when there exist sufficiently many domains $E$ the solution in a sense of definition 6 will be also a weak solution in the sense of definition 1 .

Definition 6 in fact assumes the strategy for proving the existence theorems for conservation laws, which seems different from the method of apriori estimates. Let us 
associate with any function $\boldsymbol{U}(t, x)$ from certain Banach functional space the functional $\boldsymbol{J}$ with respect to formula (8). Consider the set of such functionals. According to definition 6 in order to find the solution to system (4) we need to find $\boldsymbol{J}$ with a lot of critical points. Thus the strategy is as follows: let us start from any function $\boldsymbol{U}(t, x)$ such that corresponding functional $\boldsymbol{J}$ has some critical trajectory, than it is necessary to find the gradual perturbation of $\boldsymbol{U}(t, x)$ (and hence of $\boldsymbol{J}$ ) in order to move to the situation with more critical points of $\boldsymbol{J}$. It can be formulated as the following problem.

PROBLEM: To find the sequential deformation $\delta \boldsymbol{U}(t, x)$ of the function $\boldsymbol{U}(t, x)$ from the suitable Banach space $B$ in order that the corresponding functional $\boldsymbol{J}$ has more and more critical points.

At present there is no any solution of the posed problem. One possible way to achieve this could be using the various forms of mountain pass theorem, which states the existence of saddle critical points, see [10], for example. At this important requirement is the fulfillment of one or another form of so called Palais-Smale condition. Palais-Smale condition in its form from [10] says that if $E(u)$ is some continuously differentiable functional on some Banach space $B$ and there exists such sequence $u_{m} \in B$ that $\left|E\left(u_{m}\right)\right| \leq$ const, $\forall m$, while $\left\|\delta E\left(u_{m}\right)\right\|_{B^{*}} \rightarrow 0$ as $m \rightarrow \infty$, then $\left\{u_{m}\right\}$ has strongly convergent subsequence.

In order to demonstrate the ways to prove assertions similar to this condition we will prove easier result for functional $\boldsymbol{I}$ (10). First, consider the function $\boldsymbol{M}(\boldsymbol{U})(t, x)$, see (10), and denote by $\boldsymbol{M}(\boldsymbol{U})_{t}(t, x)$ the partial derivative of this function in its areas of smoothness.

THEOREM 10. Suppose $\boldsymbol{U}(t, x) \in K$ and suppose the functional $\boldsymbol{I}$ is coercive on some subset $A \in C([0, T], \mathbb{R})$. Also we assume that $\boldsymbol{M}(\boldsymbol{U})_{t}$ is bounded while $|x|$ is bounded. Then the set of critical points $\left\{\chi_{i} \in A\right\}, i=1,2 \ldots$ of $\boldsymbol{I}$ such that $\left|\boldsymbol{I}\left(\chi_{i}\right)\right| \leq$ const for any $i$ has the weakly convergent subsequence in the space $C([0, T], \mathbb{R})$ and $\boldsymbol{M}(\boldsymbol{U})\left(t, \lim \chi_{i_{n}}(t)\right)$ is continuous.

PROOF. The estimate $\left|\boldsymbol{I}\left(\chi_{i}\right)\right| \leq$ const and the coerciveness of $\boldsymbol{I}$ lead to the estimate $\left\|\chi_{i}\right\|_{C} \leq$ const uniformly with respect to $i$. Then some subsequence $\chi_{i_{n}}$ converges weakly in $C$, i.e. everywhere to some function $\chi$. From (13) the criticality of $\chi_{i_{n}}$ means that $\boldsymbol{M}(\boldsymbol{U})_{i_{n}} \equiv \boldsymbol{M}(\boldsymbol{U})\left(\tau, \chi_{i_{n}}(\tau)\right)$ is continuous and $\boldsymbol{M}(\boldsymbol{U})\left(\tau, \chi_{i_{n}}(\tau)\right)_{\chi}=0$ where $\boldsymbol{M}(\boldsymbol{U})_{i_{n}}$ is smooth. In the domains of smoothness

$$
\dot{\boldsymbol{M}}(\boldsymbol{U})_{i_{n}}=\boldsymbol{M}(\boldsymbol{U})_{\tau}\left(\tau, \chi_{i_{n}}(\tau)\right)+\boldsymbol{M}(\boldsymbol{U})\left(\tau, \chi_{i_{n}}(\tau)\right)_{\chi} \dot{\chi}_{i_{n}}(\tau)=\boldsymbol{M}(\boldsymbol{U})_{\tau}\left(\tau, \chi_{i_{n}}(\tau)\right) .
$$

Hence $\dot{\boldsymbol{M}}(\boldsymbol{U})_{i_{n}}$ is uniformly bounded in the smoothness areas because of our assumptions and boundedness of $\chi_{i_{n}}$. Passing to another subsequence if necessary and keeping the same 
notation $i_{n}$ we conclude that $\boldsymbol{M}(\boldsymbol{U})_{i_{n}}$ converge to some continuous function $\boldsymbol{M}(\tau)$. Because of everywhere convergence of $\chi_{i_{n}}$ we have $\boldsymbol{M}(\boldsymbol{U})(\tau, \chi(\tau))=\boldsymbol{M}(\tau)$. The theorem is proved.

\section{CONCLUSIONS}

We present the consistent variational point of view to the theory of $1 \mathrm{D}$ systems of conservation laws, which seems to be new in this area. The approach implies the alternative methods for the proof of existence and uniqueness theorems. Such methods are based on the methods of seeking critical points of nonlinear functionals in Banach spaces and differ from conventional methods of apriori estimates or vanishing viscosity. Here we describe only the framework, main problems of the theory remain open. The established theorems only illustrate the validity of the approach. The author expects the development of the highlighted theory in the forthcoming publications.

\section{REFERENCES}

[1] Yu.G. Rykov, "On the variational approach to systems of quasilinear conservation laws", Proceedings of the Steklov Institute of Mathematics, 301, 213-227 (2018).

[2] S.N. Kruzhkov, "Kvazilinejny`e uravneniya pervogo poryadka so mnogimi nezavisimy`mi peremenny`mi”, Mat. USSR Sb., 10, 217-243 (1970).

[3] J. Glimm, "Solutions in the large for nonlinear hyperbolic systems of equations", Commun. Pure Appl. Math., 18, 697-715 (1965).

[4] A. Bressan, Hyperbolic systems of Conservation Laws: the One-Dimensional Cauchy Problem, Oxford Univ. Press, (2000).

[5] R.J. DiPerna, "Measure-valued solutions to conservation laws", Arch. Ration. Mech. Anal., 88, 223-270 (1985).

[6] B.L. Keyfitz, "Singular shocks, retrospective and prospective”, Confluentes Math., 3, 445-470 (2011).

[7] D. Serre, "Divergence-free positive symmetric tensors and fluid dynamics", Annales de l'Institut Henri Poincare, 35, 1209-1234 (2018).

[8] O. A. Oleinik, "Razry`vny`e resheniya nelinejny`x differencial`ny`x uravnenij”, Uspekhi Mat. Nauk, 12:3(75), 3-73 (1957).

[9] A.I. Volpert, "Prostranstva BV i kvazilinejny`e uravneniya”, Mat. USSR Sb., 2, 225-267 (1967).

[10] M. Struwe, Variational methods. Applications to nonlinear PDEs and Hamiltonian systems, Springer, Berlin, Heidelberg, (1990).

Received July 20, 2019. 